improves the safety of intrathecal injections and leads other countries in the same way. However more advanced scientific studies of these connectors should be published. The main line of thought should be the standardisation of these connectors. Lack of standardisation is generating some hazards and supervised implementation of these medical devices is required.

No conflict of interest.

\section{GRP-172 SELECTION AND IMPLEMENTATION OF PERFORMANCE INDICATORS MEASURING THE QUALITY OF THE CLINICAL PHARMACY SERVICE OF THE MATER MISERICORDIAE UNIVERSITY HOSPITAL}

doi:10.1136/ejhpharm-2013-000276.172

'B Ryan, 'P Ging, 'J Brown, ${ }^{2} \mathrm{R}$ Edwards, 'C Meegan. 'Mater Misericordiae University Hospital, Pharmacy Department, Dublin 7, Ireland (Rep.); 'Robert Gordon University, Pharmacy Department, Aberdeen, UK

Background The Health Information and Quality Authority (HIOA) in Ireland are currently promoting and guiding the development of key performance indicators and minimum data sets to monitor health care quality. A third of Irish hospital pharmacies surveyed in 2006 believed that performance indicators were the most effective quality assessment tool. Despite this, performance indicators for clinical pharmacy services in Ireland have not been published.

Purpose To obtain consensus on whether performance indicators identified from the literature provide a valid and feasible method of measuring the quality of the Mater Misericordiae University Hospital (MMUH) clinical pharmacy service and whether they could be introduced as a regular quality measurement.

Materials and Methods Review the literature relating to the use of performance indicators in a clinical pharmacy setting and identify performance indicators which have been piloted or used in other institutions.

Achieve consensus of a multidisciplinary panel, using a Delphi method of the most valid and feasible performance indicators for the MMUH clinical pharmacy service

Implement one of the selected performance indicators

Make recommendations on the further use of performance indicators

Results Performance indictors relating to hospital pharmacy are available $(\mathrm{n}=240)$ in the literature.

The Delphi method achieved consensus and rated the following three performance indicators as both valid and feasible:

Percentage of reserve antimicrobials checked by a clinical pharmacy for approval by microbiology or infectious diseases

Percentage of patients discharged on warfarin who receive warfarin counselling by a clinical pharmacist

Percentage of medication orders for intermittent therapy that have been reviewed by a clinical pharmacist for safe prescribing.

The indicator chosen for measurement was the percentage of medication orders for intermittent therapy that were reviewed by a clinical pharmacist for safe prescribing. A $79 \%$ compliance with this performance indicator was achieved by the clinical pharmacy service.

Conclusions A multidisciplinary panel achieved consensus that three of the performance indicators identified from the literature provide valid and feasible methods of measuring the quality of the clinical pharmacy service of the MMUH. One of these was successfully implemented and consideration will be given to implementing further performance indicators

No conflict of interest.

\section{GRP-173 SEVERE ANAEMIA CAUSED BY DRUG INTERACTION. A CASE STUDY}

doi:10.1136/ejhpharm-2013-000276.173

M Perpinya, L Mallart, D Malla, E De Puig. Institut d'Assitència Sanitària, Pharmacy, Salt (Girona), Spain

Background Retrospective study based on the clinical history and the Naranjo causality algorithm.

Purpose To describe a case of severe anaemia in a HIV-positive patient receiving zidovudine and lamotrigine

Materials and Methods A 54-year old male HIV patient on antiretroviral therapy since 2002 (zidovudine $300 \mathrm{mg} / 12 \mathrm{~h}$, lamivudine $150 \mathrm{mg} / 12 \mathrm{~h}$ and abacavir $300 \mathrm{mg} / 12 \mathrm{~h}$ ), with partial epileptic seizures treated with lamotrigine (100 mg/12 h) since May 2011 who in 2007 developed low haemoglobin and haematocrit levels. A diagnosis of macrocytic anaemia was made and the patient was followed up every six months without treatment. In July 2011, at the Drug Care Unit, very low levels of haemoglobin (RBCs $1.17 \mathrm{M} / \mathrm{mcL}$, haemoglobin $5 \mathrm{~g} / \mathrm{dL}$, haematocrit 15\% and MCV $128 \mathrm{fL}$ ), asthenia, weight loss, and dyspnoea upon exertion were detected. These findings were reported to the treating doctor and the patient was admitted, with temporary discontinuation of antiretroviral and antiepileptic treatment. While in hospital, the patient required three consecutive erythrocyte concentrate transfusions.

Results At 8 weeks post-transfusion and discontinuation of antiretroviral and antiepileptic therapy, the patient's blood levels returned to normal. Antiretroviral and antiepileptic therapy was reinitiated with different drugs.

The causality relationship between severe macrocytic anaemia and zidovudine was shown to be 'probable' using the Naranjo Algorithm. Zidovudine causes macrocytic anaemia described in the data sheet as 'frequent' (1\%). According to the lamotrigine data sheet, haematological alterations are rare $(<0.01 \%)$. In this case, the macrocytic anaemia that was probably caused by zidovudine might have been made worse by a drug that rarely presents haematological toxicity.

Conclusions Macrocytic anaemia is a common serious adverse reaction to zidovudine. This drug can also cause accumulated toxicity when administrated with drugs that may also cause haematological alterations. Patients receiving these drugs require close monitoring and coordination between physician and pharmacist.

No conflict of interest.

\section{GRP-174 SIGNIFICANCE OF POTENTIALLY INAPPROPRIATE MEDICINES FOR ELDERLY PATIENTS AT A GERMAN UNIVERSITY HOSPITAL}

doi:10.1136/ejhpharm-2013-000276.174

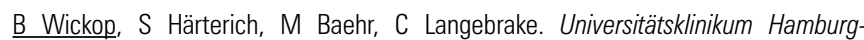
Eppendorf, Pharmacy, Hamburg, Germany

Background Certain drugs are classified as potentially inappropriate medication (PIM) for the elderly because they bear an increased risk of adverse drug events resulting in major safety concerns. Several classifications have been published to identify and avoid PIM. For this study FORTA [1] (fit for the aged), PRISCUS [2] (Latin: time-honoured) and STOPP [3] (Screening Tool of Older Persons' potentially inappropriate Prescriptions) criteria have been chosen as the most relevant ones.

Purpose The aims are to determine which PIM are taken by elderly patients at University Medical Center Hamburg-Eppendorf (UKE) and how the prevalence of PIM changes from admission to discharge

Materials and Methods Based on the criteria provided by FORTA, PRISCUS and STOPP, medication of patients $>65$ years is 